\title{
Descripción y aplicación del modelo Poisson-Pareto en el análisis de frecuencia de crecientes con series de duración parcial
}

\author{
D.F. Campos-Aranda \\ Facultad de Ingeniería de la UASLP \\ Email:dcampos@uaslp.mx
}

(recibido: enero de 1999; aceptado: abril de 1999)

\begin{abstract}
Resumen
Inicialmente, este artículo describe conceptos de tópicos asociados con el tema tratado. En seguida se expone con detalle el modelo probabilístico Poisson-Pareto, incluyendo sus bases teóricas, ecuaciones y el procedimiento de estimación de sus parámetros de ubicación, escala y forma. Posteriormente, se citan y discuten dos aplicaciones numéricas reales y se formulan varios comentarios relativos a la utilidad del modelo descrito.
\end{abstract}

Descriptores: excedencias, distribución Pareto, proceso de Poisson, Series de Duración Parcial.

\begin{abstract}
Firstly, several topics associated to this work are conceptually described. Then, the probabilistic Poisson-Pareto model is described in detail, including its theoretical bases, equations and estimation procedures for shape, scale and location parameters. Later on, two actual numerical applications are cited and discussed. Finally, several comments are advanced about the potential application of the model described.
\end{abstract}

Keywords: overflow, Pareto's distribution, Poisson's processes, partial duration series

\section{Introducción}

\section{¿Qué es el análisis de frecuencia?}

Uno de los problemas fundamentales de la hidrología superficial consiste en estimar crecientes o gastos máximos asociados con una determinada probabilidad de excedencia o riesgo de ocurrencia en un cierto lapso; estas estimaciones constituyen el objetivo fundamental de la hidrología probabilística (Campos, 1998, 1999). Con tales magnitudes se realiza el diseño hidráulico y estructural de obras de infraestructura contra crecientes, tales como: bordos o diques de protección o encauzamiento, alcantarillas y puentes, almacenamientos pequeños de detención, así como todo tipo de estructuras de drenaje urbano o agrícola (GASIR, 1996).

Cuando se dispone de información hidrométrica en el sitio del proyecto, cerca de éste o en su región geográfica, el análisis de frecuencia de crecientes (AFC) se lleva a cabo modelando probabilísticamente los registros o muestras disponibles y aplicando las técnicas del análisis regional de crecientes (Campos, 1994a, b). Si no existen tales datos, el AFC se aborda a través del estudio probabilístico de las lluvias máximas disponibles (Campos y Gómez de Luna, 1990) para construir una tormenta de diseño y posteriormente obtener el gasto máximo buscado, utilizando por ejemplo, la teoría del hidrograma unitario.

\section{¿Qué son las Series de Duración Parcial (SDP)?}

La modelación probabilística de crecientes, lluvias máximas, vientos y oleajes máximos, así como otras series de datos hidrológicos extremos, se puede llevar a cabo bajo dos enfoques; primero, usando una Serie Anual de Máximos (SAM), la cual considera únicamente el evento extremo de cada año; y segundo, a través de las SDP o de eventos superiores a un valor base o umbral (Fig. 1). 


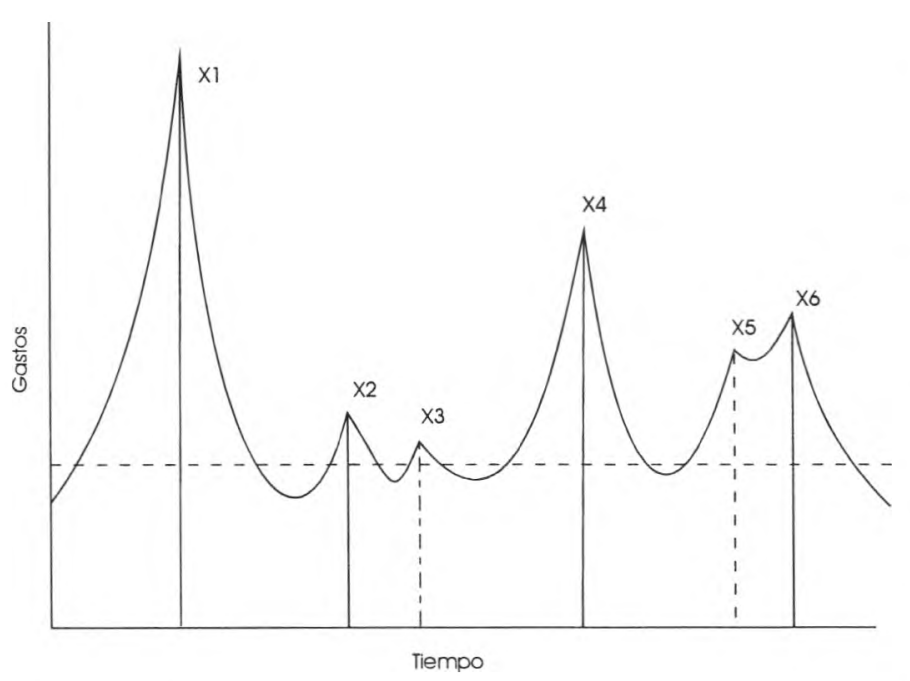

Figura 1. Representación esquemática de un hidrograma $y$ sus excedencias con respecto a un valor umbral xo

La objeción para usar la SAM es que está integrada sólo por los eventos máximos de cada año, ignorando las magnitudes secundarias que puedan exceder de las mayores de otros años. Adicionalmente, en climas áridos y semiáridos los gastos máximos anuales pueden ser de ceros o muy pequeños, de manera que es un error designar como crecientes a dichos eventos (Stedinger et al., 1993).

El análisis probabilístico de las SDP, evita los problemas anteriores al tomar en cuenta todos los eventos máximos independientes que exceden a un valor umbral; esto constituye su desventaja, ya que se debe tener un criterio para identificar solamente magnitudes independientes (Fig. 1); entonces, el análisis con SDP es un poco más complicado que el realizado con la SAM.

Otra ventaja de las SDP consiste en permitir hacer un mejor uso estadístico de los registros cortos; por ejemplo, los que no exceden de 20 años. En términos estadísticos, las estimaciones de gastos máximos procedentes de las SDP, presentan una variancia menor que las obtenidas con SAM, siempre y cuando el número de eventos exceda de 1.65 veces el número de años de registro (NRCC, 1989; Stedinger et al., 1993).

\section{¿Cómo se forman las SDP?}

Aunque en la práctica, las SDP son formadas o integradas de una manera un tanto subjetiva, en el caso de crecientes, el NERC (1975) indica que cuando dos o más gastos pico ocurren muy próximos, se adopta el mayor y del resto sólo aquellos que cumplen con las dos condiciones siguientes: a) independencia en la descarga: el descenso entre dos gastos pico debe ser al menos dos tercios del primer pico; y b) independencia en tiempo: dos gastos picos deben estar separados por al menos tres veces el tiempo de pico, éste último definido como el promedio de los tiempos de pico de 5 hidrogramas de crecientes aisladas. En el caso de lluvias máximas, Schulz (1976) indica que con dejar un lapso de dos semanas entre eventos, se garantiza que éstos procedan de fenómenos hidrometeorológicos diferentes.

Otro aspecto importante de las SDP es el número promedio de excedencias por año, recomendándose con fines prácticos que su número fluctúe de tres a cinco (Kottegoda, 1980; Taesombut y Yevjevich, 1978). Sin embargo, para asegurar la condición de independencia entre eventos, se ha recomendado, según Ashkar y Rousselle (1987), que solamente ocurran dos a tres excedencias por año.

\section{Descripción del modelo Poisson-Pareto}

\section{Aspectos conceptuales}

Dos tópicos deben ser abordados al analizar probabilísticamente SDP; el primero corresponde a la velocidad de ocurrencia de eventos mayores que el valor umbral $\left(\mathrm{x}_{\mathrm{o}}\right)$, lo cual comúnmente es modelado a través de la distribución de probabilidades de Poisson, misma que origina un proceso estocástico con igual designación.

En este punto conviene recordar que un proceso estocástico es un sistema que se desarrolla en el tiempo mientras que pasa por fluctuaciones al azar; cuando los cambios de estado ocurren en puntos aleatorios del tiempo y éstos son independientes, de manera que la probabilidad de que ocurra un evento en un intervalo $\mathrm{dt}$ es $\lambda^{*} \mathrm{dt}$, siendo $\lambda$ el número promedio de eventos que suceden en la unidad de tiempo, se tiene un proceso de Poisson. La probailidad de que ocurran exactamente $\mathrm{n}$ eventos durante un intervalo dado de longitud t, está dada por la distribución de Poisson (Maisel, 1973; Benjamin y Cornell, 1970):

$$
P_{n}(t)=\left[(\lambda t)^{n} \exp (-\lambda t)\right] / n !
$$

el número promedio de eventos que suceden durante el intervalo $t$, así como su variancia es $\lambda t$.

Esta propiedad ha sido utilizada (Ashkar y Rousselle, 1987; NRCC, 1989) para probar la aceptación del proceso de Poisson. Sin embargo, se ha encontrado que para eventos con períodos de retorno grandes, el modelo probabilístico de las ocurrencias no es importante, de manera que distribuciones diferentes conducen al mismo número promedio de eventos u ocurrencias por año (Taesombout y Yevjevich, 1978; Stedinger et al., 1993).

El segundo tópico, es el relativo al modelado de la magnitud de las excedencias, lo cual en el inicio de los 
análisis de SDP, se realizó con base en la distribución exponencial (Shane y Lynn, 1964; NERC, 1975; Kottegoda, 1980; Ashkar y Rousselle, 1987 y NRCC, 1989).

La Distribución Pareto Generalizada (DPG) incluye a las funciones exponencial y uniforme como casos particulares; además, sus propiedades y relación con la distribución o modelo General de Valores Extremos (GVE) la convierten en una selección consistente para el análisis probabilístico de SDP, como se detalla a continuación (Metcalfe, 1997).

Las dos propiedades de la DPG que la justifican como una selección razonable para el análisis de SDP son: a) si una variable aleatoria $X$ tiene DPG, la distribución condicional de $\mathrm{X}-\mathrm{x}_{0}$, también tiene una DPG con el mismo parámetro de forma $k$, cuando $X \geq x_{0}$; y b) la distribución del máximo de $\mathrm{N}$ variables con DPG es una GVE, con el mismo valor de $\mathrm{k}$, si $\mathrm{N}$ es un número aleatorio tomado de una distribución Poisson (Metcalfe, 1997).

Lo anterior significa, que en una creciente anual que es el máximo de un número de crecientes originadas por las tormentas que ocurren durante el año, y esas ocurrencias de estas crecientes son modeladas por un proceso de Poisson con magnitudes consideradas independientes, la adopción de una DPG para los valores de los gastos sobre una magnitud umbral $\mathrm{x}_{0}$, es consistente con el uso de la distribución GVE en la SAM (Metcalfe, 1997).

\section{Ecuaciones de las distribuciones GVE y Pareto generalizada}

Las expresiones de las funciones de distribución de probabilidades acumuladas $[\mathrm{F}(\mathrm{x})]$ y de la solución inversa en la DPG son (Jenkinson, 1969; Pickands, 1975; Van Montfort y Witter, 1986; Hosking y Wallis, 1987; Stedinger et al., 1993; Metcalfe, 1997):

$$
\begin{gathered}
F(x)=\exp \left\{-[1-k(x-\xi) / \alpha]^{1 / k}\right\} \\
F(x)=1-[1-k(x-\xi) / \alpha]^{1 / k} \\
x=\xi+(\alpha / k)\left\{1-[1-F(x)]^{k}\right\}
\end{gathered}
$$

$\xi, \alpha$ y k son los parámetros de ubicación, de escala y de forma. En la ecuación 3 , cuando $\mathrm{k} \leq 0$, x varía de $\xi$ a infinito y si $\mathrm{k}>0$, x fluctúa de $\xi$ a $\alpha / \mathrm{k}$. La función de densidad de probabilidad $f(x)$ para $k \neq 0$ en la DPG es:

$$
f(x)=(1 / \alpha)[1-k(x-\xi) / a]^{1 / k-1}
$$

con $\mathrm{x}$ variando de $\xi$ a $\alpha / \mathrm{k}$ o a infinito. Si $\mathrm{k}=0$ se obtiene la distribución exponencial:

$$
f(x)=(1 / \alpha) \exp [-(x-\xi) / \alpha]
$$

con $x$ fluctuando de $\xi$ a infinito. Si k excede de $1 / 2$, la DPG tiene límite superior, con un caso especial en $\mathrm{k}=1$ que es la distribución uniforme en el intervalo $[\xi, \alpha]$. Para la mayoría de las aplicaciones prácticas, $\mathrm{k}$ varía de - 1/2 a 1/2 (Metcalfe, 1997).

\section{Ecuaciones del modelo Poisson-Pareto}

Si en una SDP, $\lambda$ es el número promedio de ocurrencias o eventos por año mayores que un valor umbral $x_{0}$, y $G(x)$ la probabilidad de tener eventos, cuando éstos ocurren menores que $\mathrm{x}$, es decir, en el intervalo de $\mathrm{x}_{\mathrm{o}}$ a $\mathrm{x}$; entonces el promedio de ocurrencias para el nivel x, será (Stedinger et al., 1993):

$$
\lambda^{*}=\lambda[1-G(x)]
$$

Si $F_{a}(x)$ es la probabilidad de no excedencia en una SAM, entonces será igual a la probabilidad de no tener excedencias de $\mathrm{x}$ durante un año, la cual según la distribución Poisson será:

$$
F_{a}(x)=\exp \left(-\lambda^{*}\right)=\exp \{-\lambda[1-G(x)]\}
$$

Suponiendo que una DPG (ecuación 3) describe a $G(x)$, o sea, el comportamiento probabilístico de las magnitudes de los eventos mayores que el valor base o umbral $\mathrm{x}_{\mathrm{o}}$, entonces su sustitución en la ecuación 8 conduce a la distribución GVE para valores $\mathrm{x}$ de la SAM superiores a $\mathrm{x}_{\mathrm{o}}$, esto es:

$$
\mathrm{F}_{\mathrm{a}}(\mathrm{x})=\exp \left\{-\left[1-\mathrm{k}(\mathrm{x}-\xi) / \alpha^{*}\right]^{1 / k}\right\}
$$

Los parámetros transformados están definidos por las expresiones siguientes (Stedinger et al., 1993):

$$
\begin{gathered}
\xi=\mathrm{x}_{\mathrm{o}}+\alpha(1-\lambda-\mathrm{k}) / \mathrm{k} \\
\alpha^{*}=\alpha \lambda^{-\mathrm{k}}
\end{gathered}
$$

Las ecuaciones 9,10 y 11 constituyen el modelo Poisson-Pareto, cuyas características de flexibilidad y propiedades estadísticas lo convierten en una selección muy conveniente para modelar probabilísticamente SDP, con la ventaja adicional de que las estimaciones regionales del parámetro de forma $\mathrm{k}$ de la distribución GVE de SAM, puede ser utilizado en la ecuación 9 y viceversa (Stedinger et al., 1993; Metcalfe, 1997).

\section{Estimación de parámetros mediante momentos L}

Hosking y Wallis (1987) demostraron mediante simulación en computadora que las estimaciones de los parámetros de 
DOI: http://dx.doi.org/10.22201/fi.25940732e.2000.01n5.021

escala y forma $(\alpha, \mathrm{k})$ de la DPG, mediante los métodos de momentos y momentos de probabilidad pesada, son más confiables que las obtenidas a través del criterio de máxima verosimilitud, al menos en muestras menores de 500 elementos. Siendo los momentos L, una combinación lineal de los momentos de probabilidad pesada ( $\beta)$, los métodos son equivalentes. Stedinger et al., (1993) presentan las ecuaciones para estimar los parámetros de forma y escala del modelo Poisson-Pareto, según el método de los momentos L; éstas son:

$$
\begin{aligned}
& \mathrm{k}=\left[\left(\lambda_{1}-\mathrm{x}_{0}\right) / \lambda_{2}\right]-2 \\
& \alpha=\left(\lambda_{1}-\mathrm{x}_{0}\right)(1+\mathrm{k})
\end{aligned}
$$

siendo $\lambda_{1}$ y $\lambda_{2}$ el primero y segundo momentos $L$, cuyas expresiones, en función de los momentos de probabilidad pesada $\beta_{0}$ y $\beta_{1}$ son:

$$
\begin{gathered}
\lambda_{1}=\beta_{0} \\
\lambda_{2}=2 \beta_{1}-\beta_{0}
\end{gathered}
$$

con:

$$
\begin{gathered}
\beta_{0}=(1 / n) \sum_{1}^{n} x_{j}=X m \\
\beta_{1}=\{1 /[n(n-1)]\} \sum_{1}^{n-1}(n-j) x_{j}
\end{gathered}
$$

$\mathrm{x}_{\mathrm{j}}$ son los datos ordenados en forma decreciente y $\mathrm{n}$ el número total de datos. Lógicamente, $\mathrm{Xm}$ es la media aritmética.

La sustitución de los valores obtenidos con las ecuaciones 12 y 13 , en las expresiones 10 y 11 , definen las magnitudes de los parámetros de ubicación, escala y forma que se aplican en la ecuación 4 para obtener las estimaciones asociadas a una determinada probabilidad de no excedencia $\mathrm{F}(\mathrm{x})$, cuya relación con el lapso de retorno $\mathrm{Tr}$ de diseño (GASIR, 1996), está dada por la expresión siguiente:

$$
\operatorname{Tr}=1 /[1-\mathrm{F}(\mathrm{x})]
$$

\section{Bondad de ajuste (contraste numérico)}

Con la idea de hacer compatible la medición de la bondad de ajuste que se logra con los modelos probabilísticos al procesar una SAM, con el análisis expuesto para las SDP, se debe seleccionar el valor umbral $x_{0}$ de manera que el número de excedencias $M$, sea similar al número de años de registro (n). Esta limitante impuesta a las SDP, cuando menos permite excluir los eventos anuales de magnitud reducida, que tal vez proceden de condiciones meteorológicas diferentes a las que generan las grandes crecientes (Hosking y Wallis, 1987). Como medida cuantitativa de tal bondad de ajuste se utilizará el error estándar medio (EEM), definido por Kite (1977) como:

$$
\mathrm{EEM}=\left[\sum_{1}^{n}\left(\mathrm{Xob}_{\mathrm{i}}-\mathrm{XTr}_{\mathrm{i}}\right)^{2} /(\mathrm{n}-\mathrm{np})\right]^{1 / 2}
$$

donde, np es el número de parámetros de ajuste de los modelos probabilísticos utilizados. En el análisis de las SDP, $n p=3$ y $n=M$, o número de excedencias que ocurren, es decir, $n * \lambda$. $\mathrm{Xob}_{i}$ son las excedencias observadas ordenadas de mayor a menor y $\mathrm{XTr}_{\mathrm{i}}$ son las magnitudes estimadas con la ecuación 4 para los mismos períodos de retorno asignados a las excedencias, con base en la ecuación siguiente:

$$
\operatorname{Tr}=M / i
$$

\section{Bondad de ajuste (contraste gráfico)}

En el inicio de los análisis de SDP, se utilizó un papel semilogarítmico para dibujar las excedencias y contrastar gráficamente el modelo lineal utilizado (Shane y Lynn, 1964; Campos, 1993). Ahora, al hacer uso de un modelo más flexible o versátil, es conveniente dibujar excedencias y modelo en papeles de probabilidad; por ejemplo, Lognormal y Extremo o de Gumbel-Powell (Chow, 1964). Partiendo de la ecuación 8 se obtiene la expresión que define la probabilidad de no excedencia $G(x)$ de cada evento de la SDP y su correspondiente probabilidad de excedencia $Q(\mathrm{x})$ :

$$
\mathrm{G}(\mathrm{x})=1-\mathrm{Q}(\mathrm{x})=1+\operatorname{Ln}\left[\mathrm{F}_{\mathrm{a}}(\mathrm{x})\right] / \lambda
$$

en la cual, $F_{a}(x)$ es la probabilidad de no excedencia en la SAM y por lo tanto se puede estimar con la fórmula de Weibull:

$$
F_{a}(x)=P(X \leq x)=i /(M+1)
$$

con i variando de 1 a M cuando las excedencias han sido ordenadas de menor a mayor. El tiempo de retorno respectivo de cada excedencia o evento de la SDP (Tp) será (Stedinger et al., 1993):

$$
\mathrm{Tp}=1 /[\lambda * \mathrm{Q}(\mathrm{x})]
$$




\section{Aplicaciones numéricas}

Río Coquet en Rothbury, Northumberland, Inglaterra

En la tabla 1 se muestran los 485 datos de gastos medios diarios $\left(\mathrm{m}^{3} / \mathrm{s}\right)$ durante 21 años de registro $(1973-1993)$, los cuales son considerados independientes ya que se siguió el criterio expuesto en el inciso sobre ¿Cómo se forman las SDP? (NERC, 1975), modificado sensiblemente, pues se utilizó un mínimo de 3 días entre gastos pico. Además, sólo se consideraron los valores superiores a la media del registro y la depresión o "valle" entre picos subsecuentes menor de $2 / 3$ del primero (Metcalfe, 1997).
Los resultados para valores umbral $\left(\mathrm{x}_{\mathrm{o}}\right)$ diferentes, tanto de los parámetros de la DPG, como de las estimaciones asociadas a espacios de retorno de diseño de 10 , 100 y 1000 años se presentan en la tabulación siguiente. Esta aplicación numérica se realizó para verificar el funcionamiento correcto del programa de cómputo desarrollado.

\begin{tabular}{rrccccccc}
\hline $\begin{array}{c}\mathrm{X}_{\mathrm{o}} \\
\mathrm{m}^{3} / \mathrm{s}\end{array}$ & $\mathrm{M}$ & $\lambda$ & $\begin{array}{c}\xi \\
\mathrm{m}^{3} / \mathrm{s}\end{array}$ & $\begin{array}{c}\alpha^{*} \\
\mathrm{~m}^{3} / \mathrm{s}\end{array}$ & $\mathrm{k}$ & $\begin{array}{c}\mathrm{X}_{10} \\
\mathrm{~m}^{3} / \mathrm{s}\end{array}$ & $\begin{array}{c}\mathrm{X}_{100} \\
\mathrm{~m}^{3} / \mathrm{s}\end{array}$ & $\begin{array}{c}\mathrm{X}_{1000} \\
\mathrm{~m}^{3} / \mathrm{s}\end{array}$ \\
\hline 60 & 25 & 1.190 & 64.181 & 24.490 & -0.243 & 139.7 & 271.9 & 503.1 \\
50 & 37 & 1.762 & 64.815 & 27.310 & -0.153 & 140.2 & 247.6 & 400.6 \\
40 & 51 & 2.429 & 66.382 & 30.150 & -0.032 & 138.4 & 215.8 & 299.1 \\
30 & 85 & 4.048 & 62.912 & 27.414 & -0.224 & 145.5 & 283.7 & 515.2 \\
20 & 167 & 7.952 & 61.072 & 26.664 & -0.302 & 149.8 & 327.9 & 685.2 \\
\hline
\end{tabular}

Tabla 1. 485 gastos medios diarios $\left(\mathrm{m}^{3} / \mathrm{s}\right)$ en el río Coquet en la estación hidrométrica Rothbury en Northumberland, Inglaterra. Período de registro 1973-1993 (Metcalfe, 1997)

\begin{tabular}{|c|c|c|c|c|c|c|c|c|c|c|c|c|}
\hline 23.9 & 33.8 & 22.0 & 24.6 & 9.1 & 37.8 & 34.6 & 32.1 & 7.2 & 46.2 & 9.2 & 10.8 & 13.3 \\
\hline 5.8 & 15.8 & 29.9 & 9.4 & 21.5 & 12.1 & 27.3 & 21.2 & 6.7 & 8.3 & 17.4 & 48.5 & 29.7 \\
\hline 6.4 & 7.8 & 60.2 & 7.7 & 9.1 & 44.6 & 9.5 & 12.0 & 142.5 & 10.3 & 6.4 & 10.0 & 58.8 \\
\hline 17.1 & 13.1 & 34.9 & 14.3 & 37.8 & 16.9 & 30.0 & 5.8 & 23.6 & 8.9 & 18.7 & 6.4 & 54.2 \\
\hline 7.2 & 8.5 & 25.8 & 40.2 & 43.5 & 36.3 & 16.1 & 8.2 & 6.3 & 39.3 & 8.0 & 8.7 & 18.3 \\
\hline 5.9 & 11.8 & 77.5 & 16.2 & 9.5 & 15.7 & 7.2 & 5.8 & 8.6 & 17.4 & 6.7 & 190.9 & \\
\hline 5.7 & 11.6 & 19.0 & 12.7 & 6.5 & 21.4 & 28.0 & 8.0 & 10.2 & 91.4 & 9.0 & 26.8 & \\
\hline 10.5 & 23.0 & 10.6 & 57.4 & 13.9 & 180.7 & 6.1 & 10.9 & 9.8 & 15.2 & 20.4 & 12.0 & \\
\hline 8.7 & 8.2 & 13.6 & 77.4 & 11.2 & 8.0 & 12.7 & 18.1 & 7.5 & 8.6 & 14.4 & 9.6 & \\
\hline 9.9 & 5.7 & 13.1 & 72.8 & 9.9 & 39.2 & 15.0 & 16.7 & 27.2 & 14.3 & 38.8 & 8.5 & \\
\hline 22.0 & 10.5 & 9.5 & 26.2 & 17.3 & 19.1 & 23.0 & 9.6 & 28.8 & 11.8 & 23.7 & 8.1 & \\
\hline 19.9 & 12.8 & 17.4 & 10.9 & 21.3 & 10.1 & 48.1 & 21.0 & 21.4 & 21.1 & 30.0 & 7.0 & \\
\hline 6.4 & 7.6 & 6.1 & 9.1 & 7.6 & 8.4 & 17.9 & 9.7 & 11.3 & 24.5 & 24.3 & 14.2 & \\
\hline 23.0 & 6.1 & 8.3 & 113.2 & 8.9 & 9.3 & 8.5 & 16.3 & 44.3 & 9.5 & 9.4 & 5.7 & \\
\hline 20.4 & 20.2 & 11.3 & 101.7 & 16.3 & 10.1 & 21.6 & 10.3 & 37.1 & 6.9 & 11.4 & 11.5 & \\
\hline 19.0 & 15.8 & 13.4 & 21.1 & 19.1 & 11.5 & 8.9 & 11.7 & 51.3 & 6.4 & 6.4 & 35.1 & \\
\hline 17.1 & 8.4 & 7.6 & 56.0 & 21.8 & 9.1 & 19.3 & 29.9 & 12.4 & 21.0 & 7.7 & 7.4 & \\
\hline 18.0 & 6.0 & 17.9 & 85.2 & 8.3 & 7.7 & 86.6 & 7.4 & 18.1 & 9.5 & 14.7 & 8.4 & \\
\hline 25.4 & 6.8 & 13.1 & 28.1 & 32.6 & 33.7 & 19.5 & 23.6 & 22.9 & 6.3 & 13.4 & 15.6 & \\
\hline 13.9 & 14.8 & 18.9 & 8.0 & 15.4 & 18.9 & 9.5 & 8.0 & 10.6 & 23.0 & 7.8 & 14.5 & \\
\hline 23.8 & 16.1 & 27.0 & 12.9 & 6.1 & 10.2 & 13.6 & 8.1 & 10.4 & 25.8 & 13.7 & 30.2 & \\
\hline 6.7 & 27.0 & 7.1 & 6.7 & 9.1 & 11.4 & 7.4 & 29.0 & 37.8 & 6.0 & 25.2 & 15.0 & \\
\hline 10.3 & 25.4 & 6.8 & 17.1 & 13.2 & 17.7 & 76.4 & 20.3 & 17.5 & 5.7 & 8.4 & 32.3 & \\
\hline 14.9 & 10.0 & 11.4 & 9.3 & 5.8 & 28.3 & 6.3 & 11.4 & 29.9 & 15.9 & 17.0 & 6.0 & \\
\hline 7.5 & 18.2 & 66.2 & 10.3 & 11.3 & 30.2 & 7.1 & 33.1 & 57.0 & 36.2 & 27.1 & 9.0 & \\
\hline 7.3 & 6.2 & 16.7 & 15.8 & 117.1 & 16.9 & 7.2 & 16.4 & 15.1 & 25.6 & 62.5 & 13.0 & \\
\hline 9.4 & 13.7 & 89.6 & 10.9 & 49.4 & 25.5 & 81.3 & 32.1 & 8.0 & 9.2 & 30.4 & 53.1 & \\
\hline 15.4 & 10.4 & 25.4 & 30.8 & 19.8 & 21.1 & 19.6 & 13.1 & 5.9 & 57.9 & 15.9 & 29.4 & \\
\hline 7.8 & 23.3 & 8.5 & 25.3 & 12.3 & 9.6 & 35.8 & 14.8 & 7.6 & 25.8 & 28.7 & 10.1 & \\
\hline 14.6 & 6.6 & 10.0 & 19.4 & 23.7 & 17.8 & 25.9 & 13.8 & 25.1 & 7.4 & 7.0 & 7.1 & \\
\hline 12.2 & 31.5 & 8.4 & 17.9 & 5.8 & 8.5 & 11.6 & 10.2 & 5.7 & 5.8 & 106.8 & 13.1 & \\
\hline 54.7 & 47.5 & 12.5 & 17.0 & 24.4 & 12.2 & 12.1 & 66.3 & 24.4 & 9.0 & 15.9 & 63.8 & \\
\hline 25.7 & 9.9 & 15.6 & 39.4 & 7.5 & 13.1 & 5.8 & 10.8 & 55.7 & 6.9 & 31.6 & 21.4 & \\
\hline 7.3 & 63.9 & 11.6 & 13.4 & 6.4 & 13.1 & 11.0 & 11.6 & 5.7 & 16.2 & 11.9 & 13.8 & \\
\hline 8.6 & 27.8 & 6.7 & 8.5 & 7.1 & 23.2 & 6.5 & 7.3 & 5.9 & 23.0 & 27.7 & 58.4 & \\
\hline 9.0 & 17.7 & 8.6 & 10.1 & 12.9 & 17.5 & 12.0 & 63.1 & 42.1 & 23.2 & 6.4 & 20.2 & \\
\hline 17.4 & 13.7 & 9.4 & 17.5 & 29.7 & 8.0 & 47.2 & 32.3 & 49.8 & 15.3 & 23.7 & 6.3 & \\
\hline 20.3 & 8.5 & 6.5 & 18.5 & 10.3 & 13.9 & 6.1 & 21.0 & 13.5 & 9.0 & 7.5 & 25.8 & \\
\hline 10.0 & 37.5 & 6.4 & 66.3 & 46.7 & 32.2 & 8.2 & 7.4 & 21.0 & 6.7 & 8.4 & 71.7 & \\
\hline 41.5 & 30.9 & 7.4 & 34.2 & 54.7 & 22.6 & 26.4 & 5.8 & 9.0 & 9.7 & 23.5 & 17.2 & \\
\hline
\end{tabular}


Con respecto a los resultados anteriores, Metcalfe (1997) comenta que la dispersión observada en los resultados es reflejo de la falta de precisión en el procedimiento de estimación y, probablemente procede del cambio en la variabilidad que experimenta cada serie de excedencias conforme varía el valor umbral $\left(\mathrm{x}_{\mathrm{o}}\right)$, la cual puede estar relacionada con la estacionalidad de las crecientes. Por ello, es imprescindible realizar tal análisis de sensibilidad para observar la magnitud de los cambios en los resultados. Además, se recomienda realizar un contraste gráfico de resultados; por ello y con fines ilustrativos, en la figura 2 se han dibujado los datos y el modelo Poisson-Pareto para el valor umbral de $60 \mathrm{~m}^{3} / \mathrm{s}$, en papel de probabilidad Log-normal.

Río Valles en la estación hidrométrica Santa Rosa, San Luis Potosí

La estación de aforos Santa Rosa drena un área de $3521 \mathrm{~km}^{2}$ y se ubica a escasos $5 \mathrm{~km}$ al noroeste de ciudad Valles, pertenece a la región hidrológica No. 26 parcial (cuenca baja del río Pánuco), en la cuenca del río Tampaón (SRH, 1968). El período de registro disponible abarca de mayo de 1958 a diciembre de 1996, con los años de 1982 y 1983 faltantes, por lo tanto $\mathrm{n}=37$. Los gastos máximos instantáneos mensuales que se presentan en la tabla 2, proceden del sistema BANDAS (CNA, 1997), y tienen dos particularidades; la primera, que son las excedencias de $250 \mathrm{~m}^{3} / \mathrm{s}$ y la segunda, que cumplen con un lapso mínimo de una semana entre eventos. Los resultados de su procesamiento probabilístico como SDP, con base en el modelo Poisson-Pareto se han concentrado en la tabulación siguiente:

\begin{tabular}{cccccccccc}
\hline $\begin{array}{c}\mathrm{X}_{\mathrm{o}} \\
\mathrm{m}^{3 / \mathrm{s}}\end{array}$ & $\mathrm{M}$ & $\lambda$ & $\begin{array}{c}\xi \\
\mathrm{m}^{3 / \mathrm{s}}\end{array}$ & $\begin{array}{c}\alpha^{*} \\
\mathrm{~m}^{3 / s}\end{array}$ & $\mathrm{k}$ & $\begin{array}{c}\mathrm{EEM} \\
\mathrm{m}^{3} / \mathrm{s}\end{array}$ & $\begin{array}{c}\mathrm{X}_{10} \\
\mathrm{~m}^{3 / s}\end{array}$ & $\begin{array}{c}\mathrm{X}_{100} \\
\mathrm{~m}^{3 / s}\end{array}$ & $\begin{array}{c}\mathrm{X}_{1000} \\
\mathrm{~m}^{3 / s}\end{array}$ \\
\hline \hline 425 & 36 & 0.973 & 409.6 & 561.9 & 0.050 & 88.0 & 1632 & 2721 & 3692 \\
375 & 39 & 1.054 & 405.0 & 569.6 & -0.056 & 68.9 & 1635 & 2715 & 3664 \\
350 & 44 & 1.189 & 430.2 & 466.1 & -0.087 & 74.9 & 1619 & 3072 & 4849 \\
300 & 53 & 1.432 & 439.4 & 401.5 & -0.192 & - & 1602 & 3409 & 6220 \\
250 & 64 & 1.730 & 435.7 & 364.1 & -0.265 & - & 1592 & 3721 & 7645 \\
\hline \hline
\end{tabular}

Tabla 2. 68 gastos máximos mensuales [ $\geq 250.0 \mathrm{~m}^{3} / \mathrm{s}$ o anuales $(*)$ ] en el río Valles en la estación hidrométrica Santa Rosa, S.L.P. Período de registro: mayo, 1958 a diciembre, 1996 (sin datos 1982 y 1983). CNA (1997)

\begin{tabular}{|c|c|c|c|c|c|c|c|}
\hline 10 julio, 1958 & $* 1176.0$ & 5 oct., 1967 & 550.0 & 13 oct., 1973 & 250.5 & 6 oct., 1986 & $* 353.3$ \\
\hline 20 sept., 1958 & 326.0 & 12 nov., 1967 & 391.0 & 5 julio, 1974 & 302.7 & 22 sept., 1987 & $* 187.0$ \\
\hline 13 oct., 1958 & 732.0 & 16 ago., 1968 & $* 769.5$ & 23 sept., 1974 & $*^{*} 1480.0$ & 3 julio, 1988 & 329.0 \\
\hline 10 junio, 1959 & 291.0 & 30 ago., 1969 & $* 1244.0$ & 15 julio, 1975 & 575.0 & 19 ago., 1988 & $* 898.0$ \\
\hline 1 oct., 1959 & *505.0 & 8 sept., 1969 & 1193.0 & 11 ago., 1975 & 341.0 & 26 ago. 1989 & $* 267.0$ \\
\hline 8 sept., 1960 & *341.5 & 27 junio, 1970 & 252.4 & 13 sept., 1975 & $* 662.0$ & 8 ago., 1990 & $* 800.0$ \\
\hline 19 junio, 1961 & $* 728.0$ & 18 julio, 1970 & 441.0 & 12 julio, 1976 & $* 2588.0$ & 2 julio, 1991 & *1163.0 \\
\hline 20 ago., 1961 & 370.0 & 7 ago., 1970 & 532.0 & 30 sept., 1976 & 288.0 & 18 sept., 1991 & 255.0 \\
\hline 28 junio, 1962 & *324.0 & 26 sept., 1970 & $* 803.0$ & 3 sept., 1977 & $* 1884.0$ & 21 julio, 1992 & $* 1272.7$ \\
\hline 3 julio, 1963 & $* 973.0$ & 29 junio, 1971 & 581.7 & 3 oct., 1977 & 250.0 & 25 junio, 1993 & 1225.4 \\
\hline 13 ago., 1963 & 250.4 & 30 sept., 1971 & *636.0 & 24 sept., 1978 & *372.2 & 8 julio, 1993 & *2025.2 \\
\hline 20 oct., 1964 & *145.2 & 18 junio, 1972 & 301.0 & 31 ago., 1979 & $* 283.4$ & 21 sept., 1993 & 1057.7 \\
\hline 8 ago., 1965 & $* 360.0$ & 29 julio, 1972 & *393.6 & 2 oct., 1980 & *161.8 & 1 oct., 1993 & 280.5 \\
\hline 27 junio, 1966 & 1187.0 & 5 ago., 1972 & 392.0 & 20 junio, 1981 & $* 289.0$ & 18 sept., 1994 & $* 233.8$ \\
\hline 12 oct., 1966 & *1691.5 & 27 junio, 1973 & 3026.8 & 15 julio, 1984 & 348.4 & 14 ago., 1995 & $* 479.9$ \\
\hline 30 ago., 1967 & 440.0 & 9 julio, 1973 & 608.0 & 15 sept., 1984 & $* 461.7$ & 24 ago., 1996 & $* 771.5$ \\
\hline 7 sept., 1967 & *912.0 & 8 ago., 1973 & $* 951.3$ & 8 julio, 1985 & *361.3 & 7 sept., 1996 & 541.0 \\
\hline
\end{tabular}

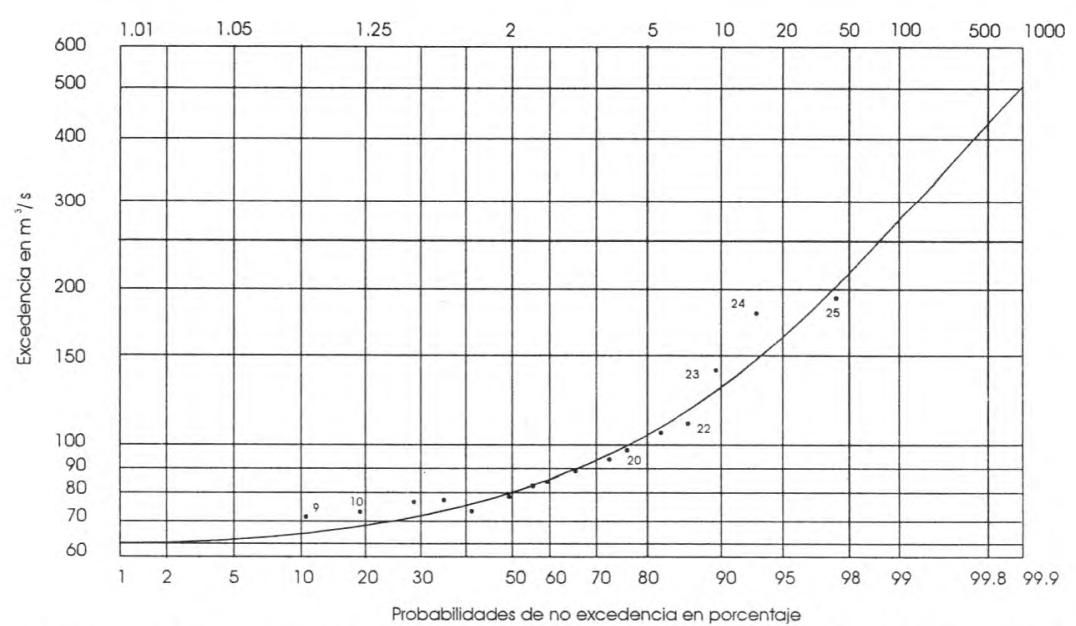

Figura 2. Distribución Poisson-Pareto ajustada a las excedencias de $x 0=60 \mathrm{~m}^{3} / \mathrm{s}$ de río Coquet (Metcalfe, 1997) 
Según se observa en tales resultados, el análisis de sensibilidad destaca una gran variabilidad, ya que el parámetro de forma de la DPG fluctúa de -0.265 a 0.050 y la estimación asociada a un lapso de retorno de 1000 años varía de 3664 $7645 \mathrm{~m}^{3} / \mathrm{s}$. El contraste gráfico de resultados se muestra en la figura 3 para el valor umbral de $350 \mathrm{~m}^{3} / \mathrm{s}$, habiéndose dibujado datos y modelo ajustado en papel Gumbel-Powell.

Por otra parte, en la tabla 2 se han destacado con asterisco los 37 valores que integran la SAM, la cual fue procesada con base en cuatro distribuciones de probabilidad: Log-Pearson tipo III (Bobée, 1975), General de valores extremos (Jenkinson, 1969; Clarke, 1973; Raynal, 1988 y Campos, 1991), Boughton (Boughton, 1980; Campos, 1996) y Wakeby (Houghton, 1978; Martínez y Raynal, 1988; Campos, 1995). Los resultados obtenidos con los cuatro modelos probabilísticos indicados fueron:

\begin{tabular}{cccccc}
\hline $\begin{array}{c}\text { Modelo probabilístico y } \\
\text { método de ajuste: }\end{array}$ & $\mathrm{np}$ & $\begin{array}{c}\mathrm{EEA} \\
\mathrm{m}^{3} / \mathrm{s}\end{array}$ & $\begin{array}{c}\mathrm{X}_{10} \\
\mathrm{~m}^{3} / \mathrm{s}\end{array}$ & $\begin{array}{c}\mathrm{X}_{100} \\
\mathrm{~m}^{3} / \mathrm{s}\end{array}$ & $\begin{array}{c}\mathrm{X}_{1000} \\
\mathrm{~m}^{3} / \mathrm{s}\end{array}$ \\
\hline $\begin{array}{c}\text { General de valores } \\
\text { extremos (momentos L) }\end{array}$ & 3 & 89.9 & 1511 & 3139 & 5599 \\
$\begin{array}{c}\text { Log-Pearson tipo III } \\
\text { (momentos dom. log.) } \\
\quad \text { Boughton }\end{array}$ & 3 & 72.1 & 1558 & 3332 & 5773 \\
\begin{tabular}{c} 
Wakeby (optimización) \\
\hline \hline
\end{tabular} & 5 & 66.9 & 1573 & 3046 & 4511 \\
\hline
\end{tabular}

Según se observa en las tabulaciones anteriores, los errores de ajuste son del orden de magnitud, con excepción del modelo Wakeby, que ha demostrado gran flexibilidad debido a sus cinco parámetros de ajuste. La comparación en las estimaciones asociadas a períodos de retorno de 10 , 100 y 1000 años son bastante consistentes en ambos análisis (SDP y SAM), lo cual brinda confianza en los valores adoptados $\left(1600,3200\right.$ y $4800 \mathrm{~m}^{3} / \mathrm{s}$, respectivamente), basados primordialmente en los resultados de la SDP con valor umbral de $350 \mathrm{~m}^{3} / \mathrm{s}$ y la distribución Wakeby. Por ello, se puede decir que tales enfoques se complementan, dando una mayor confianza a las magnitudes adoptadas, lo cual constituye el objetivo del análisis global de frecuencia de crecientes (Campos, 1999).

\section{Conclusiones}

Primero: el modelo Poisson-Pareto ofrece bases conceptuales que lo hacen recomendable para el análisis de series de duración parcial (SDP) de eventos hidrológicos extremos, comúnmente, crecientes y lluvias máximas.

Segundo: las ecuaciones que integran el método de estimación en sus tres parámetros de ajuste (ubicación, escala y forma), basado en la técnica de los momentos L son bastante sencillas de aplicar, así como la expresión que permite obtener las estimaciones asociadas a los períodos de retorno de diseño.

Tercero: el análisis de frecuencia de crecientes (AFC) con series de duración parcial permite un mejor uso estadístico de los registros hidrológicos cortos (menores de 20 años). Cuando la serie disponible es de extensión mediana (menores de 40 años), permite contar con otro enfoque de AFC, para complementar los resultados del análisis tradicional basado en la serie anual de máximos.

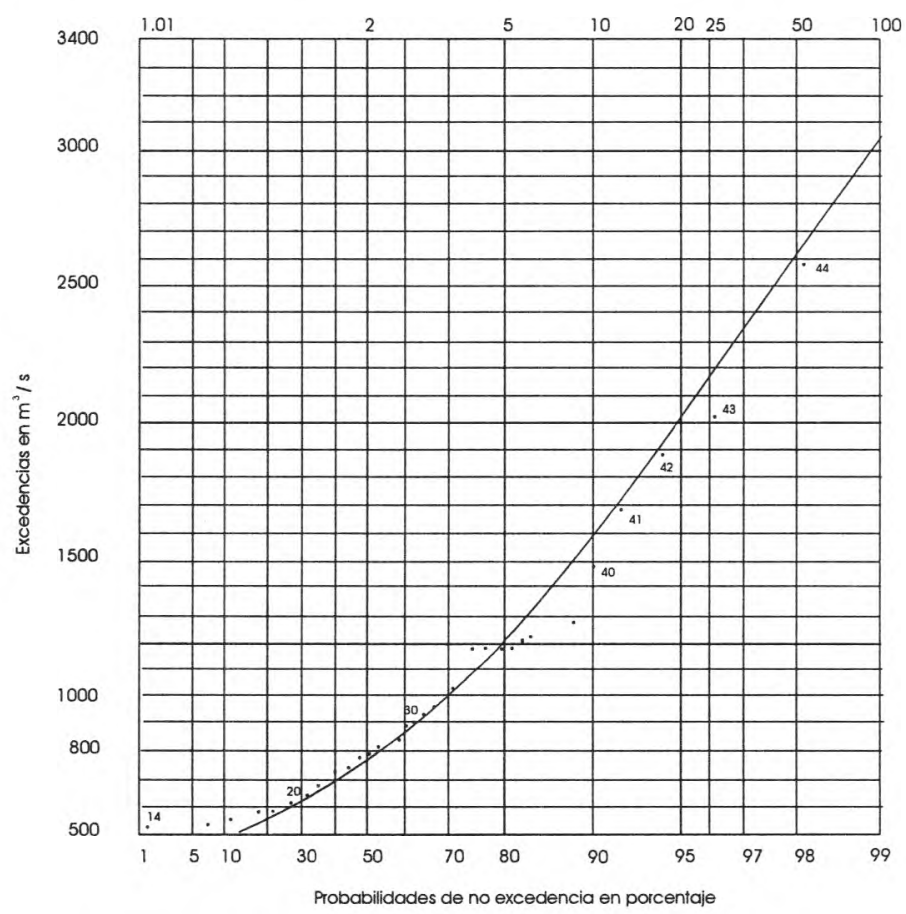

Figura 3. Distribución Poisson-Pareto ajustada a las excedencias de $x o=350 \mathrm{~m}^{3} / \mathrm{s}$ del río Valles 
DOI: http://dx.doi.org/10.22201/fi.25940732e.2000.01n5.021

\section{Agradecimientos}

Se agradece a los revisores anónimos las correcciones, comentarios y sugerencias, todos ellos fueron incorporados y contribuyeron a mejorar sustancialmente este trabajo.

\section{Referencias}

Ashkar F. y Rousselle J. (1987). Partial Duration Series Modelling Under the Assumption of a Poissonian Flood Count. Journal of Hydrology, Vol. 90, 135-144.

Benjamin J.R. y Cornell C.A. (1970). Probability, Statistics and Decision for Civil Engineers. McGraw-Hill Book Co. New York, USA.

Boughton W.C. (1980). A Frequency Distribution for Annual Floods. Water Resources Research, Vol. 16, No. 2, 347-354.

Campos-Aranda D.F. (1993). Análisis probabilístico de lluvias máximas diarias tratadas como excedencias. Informe de proyecto de investigación. Facultad de Ingeniería de la UASLP. Marzo. San Luis Potosí, S.L.P.

Campos-Aranda D.F. (1994a). Ajuste regionalizado de las distribuciones Gumbel y GVE en la región hidrológica No. 10 (Sinaloa). Método basado en los MPP. XIII Congreso Nacional de Hidráulica (AMH). Tomo II, Tema V: Investigación y Docencia, Puebla, Pue. Septiembre.

Campos-Aranda D.F. (1994b). Aplicación del método del índice de crecientes en la región hidrológica No. 10, Sinaloa. Ingeniería Hidráulica en México, Vol. IX, No. 3 (Septiembre-diciembre), 41-55.

Campos-Aranda D.F. (1995). Ajuste de la distribución de probabilidades Wakeby por medio de optimización. II Simposium Internacional de Computación. Tema II: Algoritmos, ponencia 2. Centro Nacional de Cálculo del IPN. México, DF. Noviembre.

Campos-Aranda D.F. (1996). Nuevos modelos probabilísticos para el análisis de frecuencia de crecientes. 1: Distribución de Boughton W.C. XIV Congreso Nacional de Hidráulica (AMH), Tomo I, ponencia 30. Acapulco, Guerrero. Octubre.

Campos-Aranda D.F. (1998). Hidrología probabilística. Conceptos y procedimientos. Ciencia y Desarrollo, Vol. XXIV, No. 141 (julio-agosto), 58-69. SEP-CONACYT.

Campos-Aranda D.F. (1999). Hacia el enfoque global en el análisis de frecuencia de crecientes. Ingeniería Hidráulica en México, Vol. XIV, No. 1 (enero-abril), 23-42.

Campos-Aranda D.F. y Gómez de Luna R. (1990). Procedimiento para obtener curvas I-D-Tr a partir de registros pluviométricos. Ingeniería Hidráulica en México, Vol. V, No. 2 (mayo-agosto), 39-52.
Chow V.T. (1964). Statistical and Probability Analysis of Hydrologic Data. Section 8-I, Part I: Frequency analysis, 8-42 in Handbook of Applied Hydrology, editor-in-chief Ven Te Chow. McGraw-Hill Book Co. New York, USA.

CNA: Comisión Nacional del Agua. (1997). Banco nacional de datos de aguas superficiales (BANDAS). 6 CD's. IMTA, SEMARNAP: Jiutepec, Morelos.

GASIR. Gerencia de Aguas Superficiales e Ingeniería de Ríos. (1996). Norma hidrológica que recomienda períodos de retorno para diseño de diversas obras hidráulicas. Subdirección General Técnica de la CNA. México, DF.

Hosking J.R.M. y Wallis J.R. (1987). Parameter and Quantile Estimation for the Generalized Pareto Distribution. Technometrics, Vol. 29, No. 3, 339-349.

Houghton J.C. (1978). Birth of a Parent: the Wakeby Distribution for Modeling Flood Flows. Water Resources Research 14(6):1105-1109.

Jenkinson A.F. (1969). Statistics of Extremes. Chapter 5, pp. 183-227 in the Technical Note, No. 98 (WMO-No.233): Estimation of Maximum Floods. World Meteorological Organization. Geneva, Switzerland.

Kite G.W. (1977). Frequency and Risk Analyses in Hydrology. Resources Publications. Fort Collins, CO.,USA.

Kottegoda N.T. (1980). Stochastic Water Resources Technology. The MacMillan Press Ltd. London, England.

Maisel L. (1973). Probabilidad y estadística. Fondo Educativo Interamericano, SA. Bogotá, Colombia.

Martínez H.R. y Raynal J.A. (1988). Métodos de estimación de parámetros de la distribución Wakeby. $10^{\circ}$ Congreso Nacional de Hidráulica, Tomo II. Morelia, Michoacán. Octubre.

Metcalfe A.V. (1997). Statistics in Civil Engineering. Arnold Publishers. London, England.

NRCC. National Research Council of Canada. (1989). Hydrology of Floods in Canada: A Guide to Planning and Design. Chapter 5, theme 5.2.4: Peaks over threshold (POT) approach, 54-58. Ottawa, Ontario. Canada.

NERC. Natural Environment Research Council. (1975). Flood Studies Report. Volume I, chapter 2, theme 2.7: The Peaks Over a Threshold (POT) Model, 185-210. London, England.

Pickands J. (1975). Statistical Inference Using Extreme Order Statistics. Ann. Stat., Vol. 3, 119-131.

Schulz E.F. (1976). Problems in Applied Hydrology. Water Resources Publications. Fort Collins, CO., USA.

Shane R.M. y Lynn W.R. (1964). Mathematical Model for Flood Risk Evaluation. Journal of the Hydraulics Division, Vol. 90, No. HY6, 1-20. 
SRH. Secretaría de Recursos Hidráulicos. (1968). Boletín Hidrológico, No. 32. Tomo I. Bajo Pánuco, excluyendo al río Guayalejo. Jefatura de Irrigación y Control de Ríos. México, DF. Stedinger J.R., Vogel R.M. y Foufoula-Georgiou E. (1993). Partial Duration Series, Mixtures and Censored Data, chapter 18, theme 18.37-18.42 in the Handbook of Hydrology, editor in chief David R. Maidment. McGrawHill, Inc. New York, USA.
Taesombut V. y Yevjevich V. (1978). Use of Partial Flood Series for Estimating Distribution of Maximum Annual Flood Peak. Hydrology Paper, No. 97. Colorado State University. Fort Collins, Colorado, USA.

Van Montfort M.A.J.y Witter J.V. (1986). The Generalized Pareto Distribution Applied to Rainfall Depths. Hydrological Sciences Journal, Vol. 31, No. 2, 151-162.

\section{Semblanza del autor}

Daniel Francisco Campos-Aranda. Concluyó la licenciatura de ingeniería civil en 1972 en la Escuela de Ingeniería de la UASLP. La División de Estudios de Posgrado de la Facultad de Ingeniería, UNAM le otorgó los títulos de maestro (1980) y doctor (1987) en ingeniería hidráulica y aprovechamientos hidráulicos, respectivamente. En 1989, obtuvo la medalla "Gabino Barreda" de la UNAM. Ha publicado en revistas nacionales como: Ingeniería hidráulica en México y Agrociencia. Asimismo, ha participado en congresos nacionales e internacionales. Actualmente, es investigador nacional y profesor de la División de Educación Continua en la Facultad de Ingeniería, UNAM. 